\title{
Exploring the Use of a Robust Depth-sensor-based Avatar Control System and its Effects on Communication Behaviors
}

\author{
Yuanjie $\mathrm{Wu}$ \\ HIT Lab NZ, University of Canterbury \\ Christchurch, New Zealand \\ yuanjie.wu@pg.canterbury.ac.nz
}

\author{
Yu Wang \\ Scene Simulation Lab, Beijing \\ Institute of Technology \\ Beijing, China \\ 3120160201@bit.edu.cn
}

\author{
Sungchul Jung \\ HIT Lab NZ, University of Canterbury \\ Christchurch, New Zealand \\ sungchul.jung@canterbury.ac.nz
}

\author{
Simon Hoermann \\ School of Product Design, University \\ of Canterbury \\ Christchurch, New Zealand \\ simon.hoermann@canterbury.ac.nz
}

Robert W. Lindeman

HIT Lab NZ, University of Canterbury

Christchurch, New Zealand

gogo@hitlabnz.org

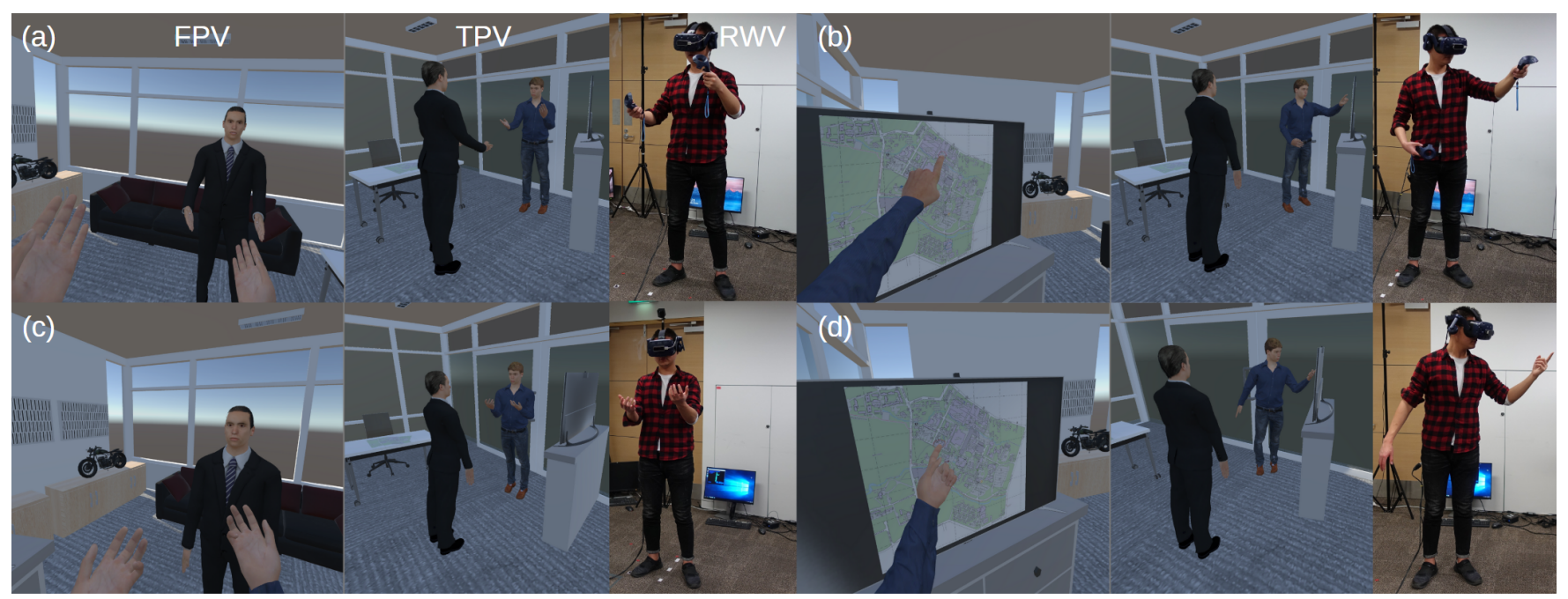

Figure 1: The virtual interview experiment in different avatar control conditions with first-person view (FPV), third-person view (TPV), and real-world view (RWV): (a) Controller-based interview session, task 1: Answer the questions, (b) Controllerbased interview session, task 2: Route planning task, (c) Depth-sensor-based interview session, task 1: Answer the questions, (d) Depth-sensor-based interview session, task 2: Route planning task

\section{ABSTRACT}

To interact as fully-tracked avatars with rich hand gestures in Virtual Reality (VR), we often need to wear a tracking suit or attach extra sensors on our bodies. User experience and performance may be impacted by the cumbersome devices and low fidelity behavior representations, especially in social scenarios where good communication is required. In this paper, we use multiple depth

Permission to make digital or hard copies of part or all of this work for personal or classroom use is granted without fee provided that copies are not made or distributed for profit or commercial advantage and that copies bear this notice and the full citation on the first page. Copyrights for third-party components of this work must be honored.

For all other uses, contact the owner/author(s).

VRST '19, November 12-15, 2019, Parramatta, NSW, Australia

(C) 2019 Copyright held by the owner/author(s).

ACM ISBN 978-1-4503-7001-1/19/11.

https://doi.org/10.1145/3359996.3364267 sensors and focus on increasing the behavioral fidelity of a participant's virtual body representation. To investigate the impact of the depth-sensor-based avatar system (full-body tracking with hand gestures), we compared it against a controller-based avatar system (partial-body tracking with limited hand gestures). We designed a VR interview simulation for a single user to measure the effects on presence, virtual body ownership, workload, usability, and perceived self-performance. Specifically, the interview process was recorded in VR, together with all the verbal and non-verbal cues. Subjects then took a third-person view to evaluate their previous performance. Our results show that the depth-sensor-based avatar control system increased virtual body ownership and also improved the user experience. In addition, users rated their non-verbal behavior performance higher in the full-body depth-sensor-based avatar system. 


\section{CCS CONCEPTS}

- Human-centered computing $\rightarrow$ Virtual reality.

\section{KEYWORDS}

depth sensor, avatar control, tracking, motion capture, communication behavior, virtual reality

\section{ACM Reference Format:}

Yuanjie Wu, Yu Wang, Sungchul Jung, Simon Hoermann, and Robert W. Lindeman. 2019. Exploring the Use of a Robust Depth-sensor-based Avatar Control System and its Effects on Communication Behaviors. In 25th ACM Symposium on Virtual Reality Software and Technology (VRST '19), November 12-15, 2019, Parramatta, NSW, Australia. ACM, New York, NY, USA, 9 pages. https://doi.org/10.1145/3359996.3364267

\section{INTRODUCTION}

Verbal and non-verbal behavior are two of the main components of communication and are essential tools for mutual understanding. Verbal communication (VC) is a direct way to express thoughts and ideas to other humans by using words, while non-verbal communication (NVC), such as body language, helps reduce the risk of misunderstandings. NVC can take many forms, such as body movements, body posture, hand gestures, eye contact (eye gaze direction, eye blinking), tone and pitch of the voice, and facial expressions.

In Virtual Reality (VR), an embodied avatar is the medium which enables people to interact and employ both VC and NVC. With modern tracking technology, NVC is generally implemented for the player through a virtual character whose behavior is captured by peripheral devices. The user can view the virtual world through the avatar's eyes, and avatar movement reflects their own body movement [Smith and Neff 2018].

At present, there are many different solutions for providing embodied virtual experiences. High-precision body tracking often comes at a high cost, and wearing devices can be cumbersome for everyday VR experiences. An alternative solution is to use controller-based avatar control with inverse kinematics (IK) methods, the accuracy of which depends on the number of sensors. The tracking quality and accuracy may impact the user's performance, body ownership, or social behavior in multi-user social scenarios, which led us to explore how different methods for controlling an embodied avatar support communication.

To understand whether different levels of expressiveness of a virtual avatar impacts communication behavior, we built an avatar control system with depth-sensor-based full body and hand tracking. To minimize the number of wearable devices while providing sufficient tracking quality, we fused the data from multiple commercial tracking sensors from four opposite directions around the user and used the data to control a virtual avatar, removing the need to wear any sensors. To explore the effects of different avatar control strategies in terms of presence, virtual body ownership, workload, usability, and communication performance in VR, we implemented a virtual interview experiment between depth-sensor-based and a controller-based avatar control approaches.

We then had subjects use both systems in a mock interview process. To assess and improve the self-evaluation experience, we went through a review session, and the user could review their previous performance in VR from a third-person point of view. The remainder of the paper describes our approach in detail.

\section{RELATED WORK}

The realism of avatars in terms of form and behavior is important for communication and collaboration in the virtual environment (VE) [Bailenson et al. 2006; Garau et al. 2003]. Most previous work has been done on visual fidelity [Latoschik et al. 2016, 2017] and the avatar appearance will influence interaction in all share VEs [Nilsson et al. 2002; Schroeder 2012]. Several researchers shared the alternative viewpoint that behavioral fidelity is a higher priority. Salinäs and Eva-Lotta [Salinäs 2002] argued that realistic appearance is secondary to support of body posture, gesture, and object manipulation in the collaboration task. Blascovich[Blascovich 2002] and Swinth[Swinth and Blascovich 2002] also argued that photographic realism is less important than behavioral realism.

\subsection{Avatar behavior representation}

Avatars are necessary to convey role, behavior, and location, etc. The behavior realism of embodied experience in VR usually requires a high-precision and real-time rendering avatar system. This helps to elicit immersive feelings in users of controllable virtual avatars as their bodies, including precise body movement tracking for hands and fingers, eyes, and even facial expressions. For the body and hand tracking, the following three ways are usually used.

2.1.1 Suit-based avatar tracking systems. A motion capture suit is a device for high quality and precise full-body tracking. Suits with reflective markers on, such as the Optitrack system [NaturalPoint 2019] are widely used in avatar-based research [Kilteni et al. 2013; Roth et al. 2016; Spanlang et al. 2014]. As the participants may have a different body size, a tracking suit is not always convenient to use. It is cumbersome for the user, and it takes time to set up. Interference [Hornung et al. 2005] from non-marker objects in the tracking area that can reflect infrared light is also another issue, which can influence the tracking quality.

2.1.2 Controller-based avatar tracking systems. The mainstream consumer VR devices such as Oculus Rift S [Facebook 2019b] with touch controllers can provide head and hand tracking with limited gestures. With a customized mapping and setting, the VIVE [HTC 2016] can also deliver an embodied experience based on the HMD and controllers. Most of the avatar-based application such as Facebook Spaces [Facebook 2019a] offers only a floating avatar (only upper body) experience because it lacks tracking data from the lower body part. Additional sensors can be used to provide key joints information and get full body tracked. Caserman et al. [Caserman et al. 2019] built the avatar tracking system using an VIVE HMD and VIVE trackers attached to the wrists and ankles to drive an avatar model in VR. The position and orientation data from the HMD and the trackers are processed before feeding into an IK system. The tradeoff is between the tracking accuracy and the number of trackers.

2.1.3 Depth sensor-based avatar tracking system. The emergence of tracking sensors such as Microsoft Kinect v2 [Microsoft 2014] and Leap Motion [Ltd 2016] appeared as Natural User Interfaces (NUI) provide a natural way for humans to communicate in a virtual 
environment. Full body and hand tracking in VR can be achieved without wearing tracking suit or extra sensors [Amir et al. 2016; Morgado et al. 2015].

\subsection{Communication behavior in VR}

The non-verbal communication behavior is usually presented in the mutual conversion through face-to-face, video conferencing [Whittaker 2003] or embodied avatar in VR [Dodds et al. 2011; Fabri et al. 2002; Roth et al. 2018; Smith and Neff 2018]. The non-verbal cues delivered by the virtual characters in the collaborative virtual environment influence the efficiency of task performance [Roth et al. 2018; Smith and Neff 2018]. The mirror is usually used in the singleuser scenario [Collingwoode-Williams et al. 2017; Kilteni et al. 2013; Maister et al. 2015] to evaluate the communication behavior such as non-verbal cue. The virtual mirror can help the user identify the consistency between their real sense and virtual representation. For those collaborative virtual scenarios, the user's social behavior and performance can be judged by another person in the shared virtual environment. Pan and Steed [Pan and Steed 2017] investigate the impact of self-avatars on trust and collaboration.

\subsection{Virtual body ownership illusion (VBOI) and agency}

Virtual body ownership illusion refers to a self-consciousness of one's own body [Gallagher 2000; Jung et al. 2018], which is a critical component to indicate the level of presence and sense of embodiment [Kilteni et al. 2012]. Yee and Bailenson found a Proteus effect that the virtual avatar's appearance and behavioral characteristic influenced to individual's behavior changes, and it depends on VBOI [Yee and Bailenson 2007]. To enhance VBOI, Gonzalez-Franco et al. and Jung et al. studied the influence of real-time behavior of the avatar using a virtual mirror that results in a higher sense of body ownership [Gonzalez-Franco et al. 2010; Jung and Hughes 2016; Jung et al. 2017]. The visuomotor is a significant factor for virtual body ownership [Blanke and Metzinger 2009; Jung et al. 2017], the freedom of agency that refers to the sensation of controlling the virtual body has been considered as an important factor for VBOI.

\section{METHODS}

We built an avatar control system based on $\mathrm{Wu}$ et al. [Wu et al. 2019]. The user can move freely with full-body (21 joints including the torso, arms, and legs) and hand gesture tracking (19 joints with pointing, grasp, and pinch). In terms of expressiveness for rich communication, we designed a study to evaluate the differences between the two experimental conditions : (1) Depth-sensor-based avatar control system: a motion-capture tracked avatar, providing an embodied representation of the user with full-body and handgesture tracking. (2) Controller-based avatar control system: using the tracked HMD and controllers to get an embodied representation with upper-body tracking and a single pointing gesture. Both conditions included a shared workplace with virtual interviewer. We will give details in the section 3.3.

\subsection{System Overview}

3.1.1 Hardware Overview. Four Kinect v2 sensors were installed on tripods placed in the corners of $3.2 \mathrm{~m} \times 3.2 \mathrm{~m}$ square tracking area.

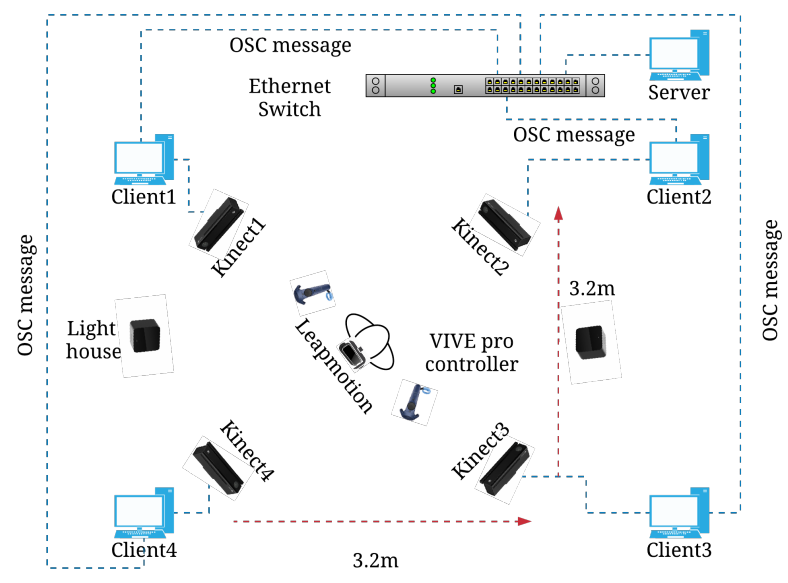

Figure 2: Setup for the depth-sensor-based avatar control system. Four Kinects tracked the user's body and Leap Motion tracked hand in real-time.

The Kinects were adjusted to a height of $1.2 \mathrm{~m}$ above the floor to avoid Infrared radiation (IR) interference between the Kinects and the HMD. Each Kinect was driven by a client machine which was an Intel NUC (Intel Core i5-8259U at $2.3 \mathrm{GHz}, 8 \mathrm{~GB}$ RAM, and Iris* Plus Graphics 655). An HTC Vive Pro with two second-generation Lighthouse sensors was driven by a server machine, which was a Windows 10 desktop computer (Intel Core i7-7700K at $4.2 \mathrm{GHz}$, 32GB RAM, and NVIDIA GeForce 1080Ti). A Leap Motion sensor was attached on the front of the HMD with a USB cable to the server. All four client machines and the server machine were connected to a Gigabit Switch (NETGEAR GS110MX) through Ethernet cables for network data transmission.

\subsubsection{Software Overview.}

- Development Tools - We developed our software using the Unity game engine version 2017.1.1f1 [Technologies 2017] with SteamVR for Unity [Corporation 2019], and Leap Motion plugin for Unity (Core 4.4.0) [Ltd 2017]. The skeleton data from each Kinect was wrapped as an Open Sound Control (OSC) message and transmitted through the network. The UniOSC plugin [Monoflow 2016] was used to handle the OSC messages received on the server machine.

- Virtual Avatar Representation - Since the sense of ownership can be induced even using a body part that is not your own [Botvinick and Cohen 1998] and our goal is not related to the personalized appearance of the avatar, we created three generic avatar models using MakeHuman software [MakeHuman 2018] in this study. Two avatars were designed for participants with standard body size, and different heights [Chapple and Ladaique 2009] for female (1.65m) and male $(1.77 \mathrm{~m})$ characters. The third character was customized with similar appearance and body size as our lab lead. To avoid the expected confounding from the facial expression since we used an interview scenario, and it could be influenced by the facial expression, we gave a plausible 
expression including mouth movement, eye movement and blinking for both conditions. We used Blender 2.79b [blender 2019] to add blend shapes on the avatars and used SALSA plugin for Unity [Studio 2014] to customize three sets of blend shapes to represent the open mouths as small, medium, or large in shape, which were triggered by the loudness of the microphone input. The eye-gaze direction was the same as the head orientation of the HMD, and the virtual avatar performed random eye blinking. We rigged the skeleton in Unity after all those procedures. The participants had different heights and body sizes. An additional calibration step was carried out to apply the predefined character to every participant. The participants were asked to stand still and make a "T-pose" before the experiment. Their height and arm length were measured and used to auto-scale the size of the virtual character.

3.1.3 Bandwidth and latency. Four client machines continuously streamed the serialized body-frame data to the server machine at a rate of 1.5Mbps over Ethernet. There were three sources of system latency: (1) Data pre-processing on the client machine. It took less than $1 \mathrm{~ms}$ for the client machine to serialize the body-frame data into an OSC message before sending it to the network once the Kinect detected the user in the tracking area. (2) Data transmission in the network. It took less than $1 \mathrm{~ms}$ for message transmission. (3) OSC message handling and avatar control rendering in the Unity game engine. The UniOSC plugin was used to process the OSC messages received on the server machine, and deserialized the data for the fusion. As this plugin works based on the game engine, it took up to $30 \mathrm{~ms}$ from data receipt and fusion to avatar rendering. Therefore, the latency of the system is less than $32 \mathrm{~ms}$ in the worst case.

\subsection{Participants}

We recruited 25 participants (13 male, 12 female) from our University through advertisements posted on campus and on University social media platforms. They were aged 18-35 $(M=26.2, S D=4.5)$. We asked participants about their familiarity with VR using a 5point Likert scale, from 1 (never), 3 (a few times a month), to 5 (daily use). The participants generally had moderate experience for using VR $(M=2.56, S D=1.19)$. Frequency of Social VR platform use was never $(72 \%)$, a few times a year (20\%), a few times a week (4\%), and daily (4\%). From the demographic information, most participants had VR experience, but only $28 \%$ of subjects had tried social VR applications before. The experiment was approved by our organization's Human Ethics Committee.

\subsection{Study Design}

We used a 1x2 within-subjects design with interview scenario in a virtual office. For each experiment condition, the participants experienced two sessions: an interview session and a review session. In the first session, we provided two specific tasks. After the participant completed a first interview session with a virtual interviewer, the participant watched their recorded interview procedure for self-evaluation purpose in the second session. We randomized the condition order using Research Randomizer [Urbaniak and Plous 1997] to avoid the ordering effect.

\subsubsection{Conditions.}

The Controller-based Avatar Control System (CB-ACS). - In this condition, the virtual character was driven by tracking the HMD and two controllers. The Final IK for Unity [RootMotion 2019] plugin was used to calculate and estimate the positions and rotations of other joints of the body excluding head, left and right hand. In this case, virtual hands and arms moved when the participants moved the controllers. The two legs of the virtual character moved automatically when the translation of the HMD changed, and the step width was adjustable. As there was no finger tracking in this condition, a pointing gesture was made when the participant pulled the trigger button.

The Depth-sensor-based Avatar Control System (DSB-ACS). - In this condition, the motion tracking data came from two parts. One was the fused skeleton, which contained 21 joints from head to feet, including the arms and legs. In each client machine, 30 frames of skeleton data retrieved from a connected Kinect. The data was serialized and sent to the server machine for 3D skeleton data fusion, which was the primary data source for body tracking. The other source was from a Leap Motion device, which was used for finger tracking and hand gesture recognition. All tracked joint data was fed into Unity for avatar control. The Leap Motion has a limited field of view $(60 \mathrm{~cm}$ vertical x $60 \mathrm{~cm}$ horizontal $\mathrm{x} 60 \mathrm{~cm}$ deep) [COLGAN 2014]. Therefore, the data for the elbows, wrists, and hands switched to the Kinect sensors whenever the hands were outside of the Leap Motion tracking area.

\subsubsection{Two sessions in this experiment.}

First session: Be an interviewee. - The effect of avatar control approach on the user's behavior can depend upon the interview questions and tasks that the participant needs to accomplish. All the interview questions and tasks were carefully designed to make sure that the participant can employ the body postures and hand gestures naturally and intuitively.

Task 1: Answering interview questions. The participant needed to answer a set of interview questions from a virtual interviewer. We adopted the tested and used interview questions by Villani et al. [Villani et al. 2012, 2017]. For each condition, three questions were adopted from the question sets. The time for each answer was two minutes. The "stop" animation and relevant audio was used to remind the user to stop and answer the next question. We prepared two interview question sets:

- Set 1: What is your greatest weakness? Where do you see yourself in five years? Tell me about a time when you used your skills of persuasion to convince someone of your ideas.

- Set 2: How will your greatest strength help you perform? What are your expectations and goals? Let us talk about your personality. What are three adjectives that best describe you?

Task 2: Route-planning Task. After the interview questions, the participant was asked to complete a route-planning task by giving directions to the interviewer while referring to a virtual map present in VR. The map was shown on a TV screen placed on a nearby cabinet. The participant had to take a few steps to get close to the TV screen. The height of the TV top to the floor was around 
$1.7 \mathrm{~m}$. In the task, the participant had to describe a path from a given starting point (red circle) to an endpoint (blue circle). We expected participants would use many social cues, such as finger or hand gestures, and body postures to confirm things with the virtual interviewer.

The non-verbal behavior of participants such as body movements, hand gestures, mouth movements, and eye blinking data was recorded at 10 frames per second. This parameter could be set at a higher level, but it consumes CPU and RAM resources, which can slow the system when storing frame data of the avatar animations. The verbal-behavior audio was recorded at a $44.1 \mathrm{kHz}$ sample rate to guarantee high quality in the review session. All the interview questions and instruction audio was recorded in advance, as well as relevant animations by a native speaker. In the interview session, the participant faced the virtual interviewer, questions and related animations were controlled manually from a researcher by pressing specific keys on the keyboard. The session for the whole process was recorded automatically in Unity. We then used the recorded time-line for the review session playback.

Second session: Review interview from third-person perspective. In this session, the participants watched their interview process through HMD. They can walk and turn around in this session to review the interview from a third-person view. The whole procedure was replayed automatically according to the recorded time-line. The participants were asked to focus on the verbal and nonverbal behavior of themselves for the following questionnaire.

3.3.3 Hypotheses. We expected better avatar controlling performance while reducing the cumbersome for holding the controller from our system. Based on the expectation, and the previous related work in the field, We formulated several hypotheses.

- $H_{1}$ : Depth-sensor-based avatar control will provide participants with a deeper sense of presence compared to controllerbased avatar control.

- $\mathrm{H}_{2}$ : Participants will feel lower mental workload when using depth-sensor-based avatar control compared to controllerbased avatar control.

- $H_{3}$ : Using the depth-sensor-based avatar control system will result in a higher sense of body ownership and agency compared to controller-based avatar control.

- $H_{4}$ : Depth-sensor-based avatar control will provide participants with a better user experience during virtual social communication compared to controller-based avatar control.

- $H_{5}$ : Participants will have better self-rated performance in terms of communication behavior by using depth-sensorbased avatar control compared to controller-based avatar control.

- $H_{6}$ : Participants will prefer to use depth-sensor-based avatar control over controller-based avatar control.

\subsection{Measurements}

Data was collected in two ways. Most subjective questionnaires were filled out after the interview session which used a first-person perspective. As the participant could move around during the review session in the virtual environment in a third-person point of view, the self-evaluation questionnaire was filled out after the review session.

3.4.1 First-person Perspective. - Dependent variables such as the sense of presence were assessed using the Igroup Presence Questionnaire (IPQ) [Schubert et al. 2001], and the sense of body ownership and agency were measured using a questionnaire about avatar embodiment [Gonzalez-Franco and Peck 2018]. As there is a route planning task, the workload was assessed by using NASA TLX [Hart and Staveland 1988]. System Usability Scale [Brooke et al. 1996] was adopted to compare the usability of the two avatar control methods.

3.4.2 Third-person Perspective. - To verify $\mathrm{H}_{5}$, participants answered the following custom questions and scored themselves (0-100) in terms of verbal and non-verbal behavior after review session.

"Think about what you saw when you watched the replay of your interview."

- (1) How realistic was your non-verbal behavior: body posture and hand gestures?

- (2) How realistic was your verbal behavior?

3.4.3 User Preference. - Finally, we created a set of post-experiment questions for comparison between depth-sensor-based and controllerbased avatar control methods in terms of ease of use and preference.

\subsection{Procedure}

After the introduction of the experiment, the participant signed the consent form and filled out the demographic survey on a laptop. The researcher explained how to use the devices involved in the study, helped the participant put on the HMD, and asked them to familiarize themselves with the controllers, and/or hand tracking devices. The participants were asked to walk around to practice how to control the virtual avatar in the two conditions. They then spent one minute looking around the virtual environment to familiarize themselves with the furniture and layout. This was done to reduce the risk of distraction during the experimental tasks.

Participants were then positioned in the center of tracking area and asked to start the condition one, session one from the firstperson point of view. The whole process was recorded and stored as animations. The audio from the participant was also recorded from the built-in microphone of Vive Pro. After the first session, the participant was asked to fill out several surveys on the laptop. In the next session (condition one, session two), the virtual camera in the scene changed to a position near the virtual interviewer. The animations and audio were loaded for replay, and the participant watched the previous interview in VR from a third-person point of view. A self-evaluation survey was filled out by the participant after the replay session. The process was repeated for the second condition.

After the second condition, participants were given one additional survey to gather information about their preference and ease of use of avatar control schemes. The researcher then performed an experimental debrief with the participant and encouraged them to write comments about the two systems, discuss their survey answers, and talk about their general impressions of the two conditions. 


\section{RESULTS}

In this section, we show the results of the effects of the depthsensor-based and controller-based avatar control approaches. For the analysis, we used 25 participant data sets. As we described in the user study section, we ran our study as $1 \mathrm{x} 2$ within-subjects design. To analyze subjective measures, paired-samples t-test was used for presence, virtual body ownership illusion (VBOI), agency, workload, usability, and self-evaluation. For significance testing, we used a confidence value of $\alpha=0.05$.

\subsection{First-person Perspective}

4.1.1 Presence and Workload. Presence was measured from the IPQ with four components: General Presence (GP), Spatial Presence (SP), Realism (REAL), and Involvement (INV), and overall workload was measured from NASA TLX. From the results are presented in Table 1 and Figure 3. We can see that there were no significant differences $(p>0.05)$ between the depth-sensor-based and controllerbased avatar control approaches.

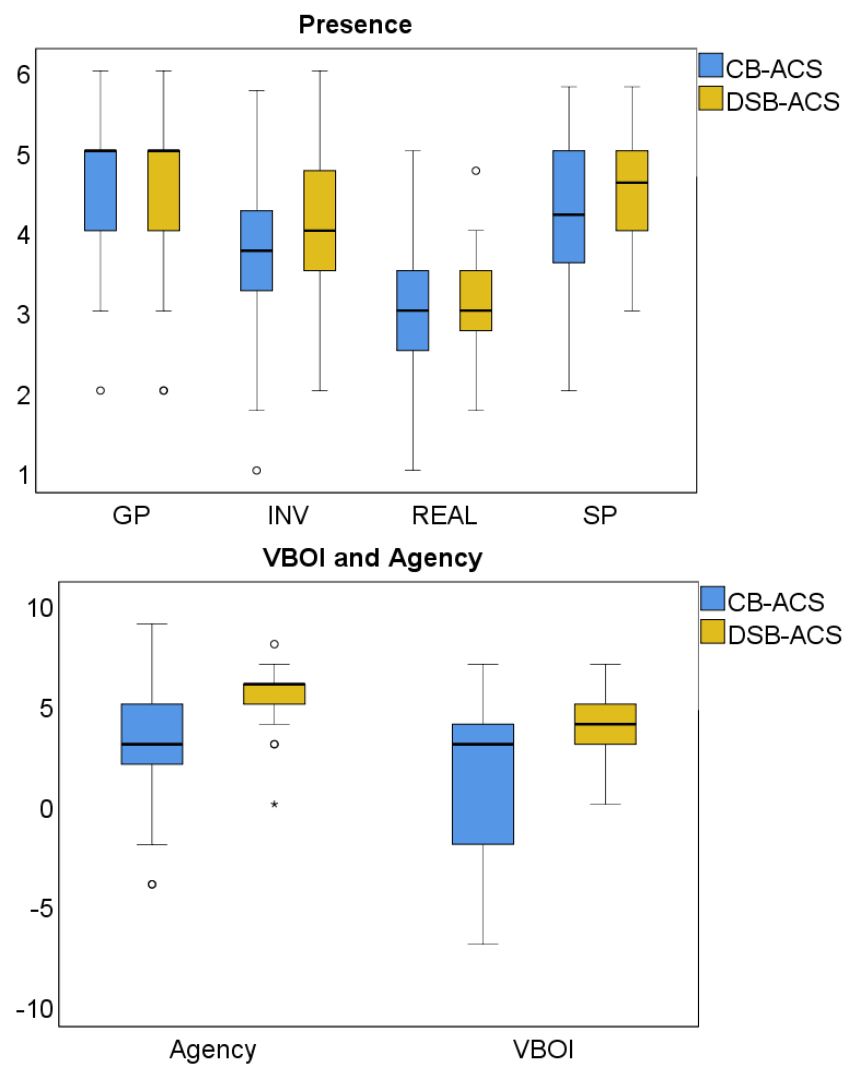

Figure 3: Presence, VBOI, and Agency

4.1.2 Virtual Body Ownership Illusion, Agency, and System Usability. The VBOI and sense of agency were measured from the avatar embodiment questionnaire. As there was no mirror placed in the virtual environment, we removed Q4 ("I felt as if the virtual__ I saw when looking in the mirror was my own__") and Q5 ("I felt as if the virtual__ I saw when looking at myself in the mirror was another person"). System usability was measured using the System Usability Scale. It is clear that there was a significant difference $(p<0.05)$ between the depth-sensor-based and controllerbased avatar control approaches in terms of VBOI $(p=0.002)$, agency $(p=0.0009)$, and usability $(p=0.0029)$ from Table 1 and Figure 3.
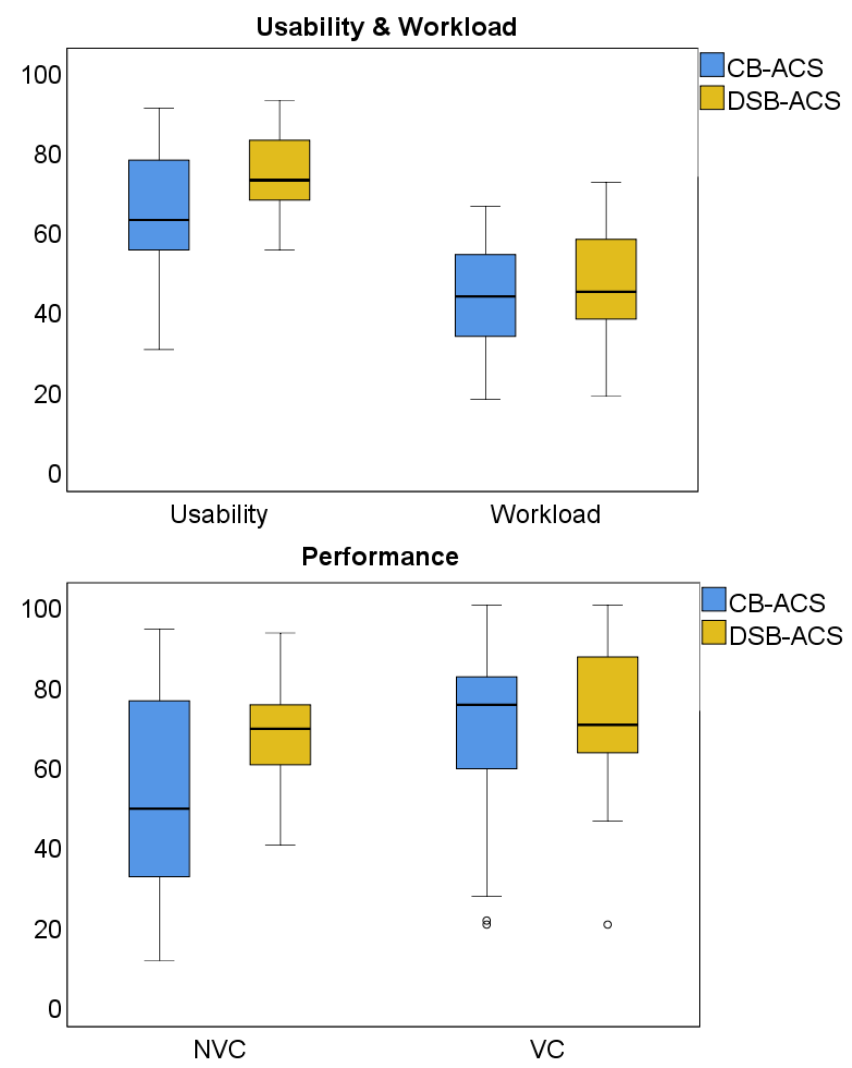

Figure 4: Usability and Workload

\subsection{Third-person Perspective}

4.2.1 Self-evaluation. From the results in Table 1 and Figure 4, we observe an interesting outcome. There was a significant difference $(p<0.05)$ between the two avatar control approaches in the interview review session in terms of non-verbal behavior $(p=0.008)$. This indicates that participants preferred realistic body posture and hand gesture while talking in the communicative scenario to improve their performance. However, there was no significant difference $(p>0.05)$ between these two conditions for verbal behavior $(p=0.37)$, because the method used to control mouth movements was the same in both approaches.

\subsection{User Preference}

The subjective opinions about ease of use and system preference can be found in Figure 5. The results show that $76 \%$ of participants thought it was easier to use depth-sensor-based avatar control approach. Furthermore, about $84 \%$ of participants preferred to use depth-sensor-based avatar control. 
Table 1: Statistical results for Presence, Workload, VBOI, Agency, Usability, and Performance

\begin{tabular}{|c|c|c|c|c|c|c|c|c|c|c|}
\hline & VBOI & Agency & Usability & \multicolumn{2}{|c|}{ Performance } & \multicolumn{4}{|c|}{ Presence } & Workload \\
\hline & & & & NVC & VC & GP & SP & INV & REAL & \\
\hline$t$-test & $p=\mathbf{0 . 0 0 2}$ & $p=\mathbf{0 . 0 0 0 9}$ & $p=\mathbf{0 . 0 0 2 9}$ & $p=\mathbf{0 . 0 0 8}$ & $p=0.37$ & $p=0.582$ & $p=0.087$ & $p=0.070$ & $p=0.215$ & $p=0.186$ \\
\hline CB-ACS $(M, S D)$ & $(1.2,4.0)$ & $(3.0,3.2)$ & $(64.1,16.2)$ & $(52.1,23.7)$ & $(68.6,23.6)$ & $(4.4,0.9)$ & $(4.2,1.0)$ & $(3.7,1.1)$ & $(2.9,1.0)$ & $(42.7,13.5)$ \\
\hline DSB-ACS $(M, S D)$ & $(3.8,2.1)$ & $(5.4,1.6)$ & $(74.0,10.6)$ & $(66.9,14.5)$ & $(72,18.6)$ & $(4.5,1.1)$ & $(4.5,0.7)$ & $(4.1,1.0)$ & $(3.2,0.7)$ & $(46.0,12.7)$ \\
\hline
\end{tabular}

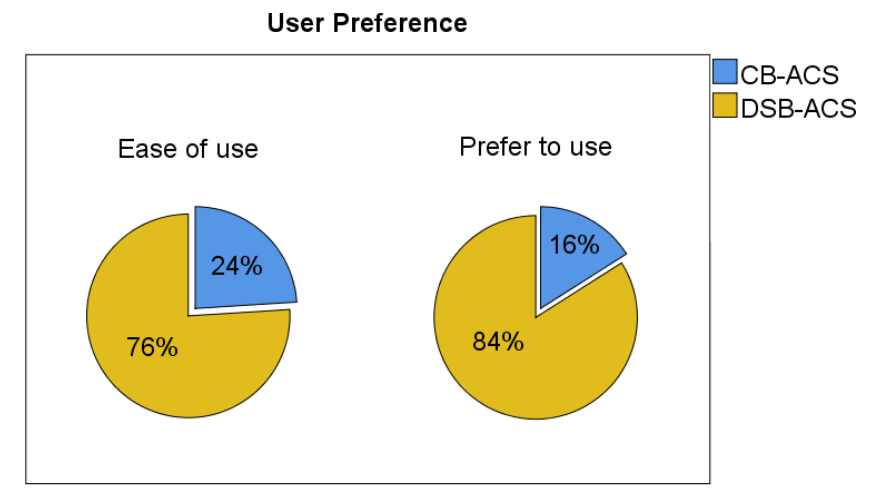

Figure 5: User Preference

The participant also gave some comments about the overall experience.

- "Depth-sensor-based avatar control is more realistic for mapping my hands in the virtual environment"

- "I believe that the depth-sensor-based avatar control system is comparatively much easier, which can give you much more freedom than the controller-based system as you might have the feeling that you are possessing something in your hands. The use of an object makes you feel that you are not truly connected to the virtual world"

- "Ifeel completely immersed in the depth-sensor-based avatar control system"

- "The depth-sensor-based system shows hand movements and finger movements more realistically and I could move my hands easier in the interview"

- "The posture looked more real in the depth-sensor-based system and tracking of arms was better too"

\section{DISCUSSION}

In this experiment, hypotheses $\mathrm{H}_{1}$ and $\mathrm{H}_{2}$ were rejected as there were no significant differences between the two avatar control systems on the sense of presence and mental workload. We did, however, find support for hypotheses $\mathrm{H}_{3}$ and $\mathrm{H}_{4}$. The subjective questionnaire responses showed a significant effect that the depthsensor-based avatar control approach elicited a higher sense of virtual body ownership illusion and agency, as well as better usability compared to the controller-based avatar control system. In support of hypothesis $\mathrm{H}_{5}$, we found that participants indicated that they had better performance in the communication scenario using the depth-sensor-based avatar control system in terms of non-verbal behavior cues, but not in terms of verbal performance between these two systems. Moreover, when asked for a preference, most participants indicated that they preferred the depth-sensor-based avatar control approach and that it was easier to use.

\subsection{Implications}

Based on these results, we would suggest that VR developers should adopt methods for the full-body tracking that are as expressive as possible. Depth-sensor-based hand tracking can provide an intuitive way for gesture-based interaction, and users do not always understand button or trigger mappings. The animation recording and reply mode provided a way to make self-evaluations from a third-person perspective. It is not only more flexible compared to inviting another person into VR, but also provides an objective way to review performance compared to facing a virtual mirror, especially for training and single-user communication simulation systems.

\subsection{Limitations}

In this user study, we found some technical limitations. First, the hand-tracking data sometimes switched between the fused Kinect system and the Leap Motion system, and there was no finger data when the participants moved their hands outside the tracked area of Leap Motion. Some participants noticed a slight hand pose change between the tracking boundaries in the interview review part. We believe that the subtle pose change did not have an impact on our experiment since only three participants reported it. Second, the first two participants reported body penetration effects while they reviewed their virtual interviews. The arms slightly penetrated the body, which was because the participants were nervous during the interview, and put their arms too close to their bodies. This occlusion issue can cause bad recognition from Kinect sensors. Therefore, from the third participant on, we asked all participants before the experiment to be relaxed and to keep some space between their arms and bodies. As these two cases only happened during the interview question part, and there was no such issue during the route-planning task, we do not think there was any impact on our results since there were no similar problems reported by the rest of the participants. Overall, we believe that none of these limitations had a noticeable effect on the results.

\section{CONCLUSIONS AND FUTURE WORK}

In this paper, we investigated the effects of a depth-sensor-based avatar control approach on presence, virtual body ownership, mental workload, usability, and communication behavior. We provided an avatar control method that supports realistic behaviors based on data from multiple depth sensors (multiple Kinects and a Leap Motion). We compared this fully-tracked body and hand-gesture avatar 
control system with a controller-based IK system as a baseline condition. We found significantly higher virtual body ownership illusion and usability as well as better non-verbal communication performance by participants in the depth-sensor-based experience compared to the controller-based experience.

In future work, we plan to integrate better hand tracking to improve the expressiveness of avatars further. Besides this, we will add more real-time capture of non-verbal cues such as eye gaze, eye blinks, and facial expressions to the avatar control and explore the effects of highly-expressive avatars on the user performance and preference in collaborative virtual environments.

\section{REFERENCES}

Mohd Hezri Amir, Albert Quek, Nur Rasyid Bin Sulaiman, and John See. 2016. DUKE: Enhancing Virtual Reality Based FPS Game with Full-body Interactions. In Proceedings of the 13th International Conference on Advances in Computer Entertainment Technology (ACE '16). ACM, New York, NY, USA, Article 35, 6 pages. https://doi.org/10.1145/3001773.3001804

Jeremy N Bailenson, Nick Yee, Dan Merget, and Ralph Schroeder. 2006. The effect of behavioral realism and form realism of real-time avatar faces on verbal disclosure, nonverbal disclosure, emotion recognition, and copresence in dyadic interaction. Presence: Teleoperators and Virtual Environments 15, 4 (2006), 359-372.

Olaf Blanke and Thomas Metzinger. 2009. Full-body illusions and minimal phenomenal selfhood. Trends in cognitive sciences 13, 1 (2009), 7-13.

Jim Blascovich. 2002. Social Influence within Immersive Virtual Environments. Springer London, London, 127-145. https://doi.org/10.1007/978-1-4471-0277-9_8

blender. 2019. blender.org - Home of the Blender project - Free and Open 3D Creation Software. https://www.blender.org/

Matthew Botvinick and Jonathan Cohen. 1998. Rubber hands 'feel' touch that eyes see. Nature 391, 6669 (1998), 756

John Brooke et al. 1996. SUS-A quick and dirty usability scale. Usability evaluation in industry 189, 194 (1996), 4-7.

Polona Caserman, Augusto Garcia-Agundez, Robert Konrad, Stefan Göbel, and Ralf Steinmetz. 2019. Real-time body tracking in virtual reality using a Vive tracker Virtual Reality 23, 2 (2019), 155-168.

Simon Chapple and Maxime Ladaique. 2009. Society at a Glance 2009: OECD social indicators. Organisation for Economic Co-operation and Development, Paris,France.

ALEX COLGAN. 2014. How Does the Leap Motion Controller Work? http://blog.leapmotion.com/hardware-to-software-how-does-the-leap-motioncontroller-work/

Tara Collingwoode-Williams, Marco Gillies, Cade McCall, and Xueni Pan. 2017. The effect of lip and arm synchronization on embodiment: A pilot study. In 2017 IEEE Virtual Reality (VR). IEEE, IEEE, Los Angeles, CA, 253-254.

Valve Corporation. 2019. steam vr. https://www.steamvr.com/en/

Trevor J Dodds, Betty J Mohler, and Heinrich H Bülthoff. 2011. Talk to the virtual hands: Self-animated avatars improve communication in head-mounted display virtual environments. PloS one 6, 10 (2011), e25759.

M Fabri, DJ Moore, and DJ Hobbs. 2002. Expressive agents: Non-verbal communication in collaborative virtual environments. Proceedings of Autonomous Agents and Multi-Agent Systems (Embodied Conversational Agents) (2002).

Facebook. 2019a. Facebook Spaces. https://www.facebook.com/spaces

Facebook. 2019b. Oculus Rift S. https://www.oculus.com/rift-s/

Shaun Gallagher. 2000. Philosophical conceptions of the self: implications for cognitive science. Trends in cognitive sciences 4, 1 (2000), 14-21.

Maia Garau, Mel Slater, Vinoba Vinayagamoorthy, Andrea Brogni, Anthony Steed, and M. Angela Sasse. 2003. The Impact of Avatar Realism and Eye Gaze Control on Perceived Quality of Communication in a Shared Immersive Virtual Environment. In Proceedings of the SIGCHI Conference on Human Factors in Computing Systems (CHI '03). ACM, New York, NY, USA, 529-536. https://doi.org/10.1145/642611.642703

Mar Gonzalez-Franco, Daniel Perez-Marcos, Bernhard Spanlang, and Mel Slater. 2010 The contribution of real-time mirror reflections of motor actions on virtual body ownership in an immersive virtual environment. In 2010 IEEE virtual reality conference (VR). IEEE, IEEE, Waltham, MA, USA, 111-114.

Mar Gonzalez-Franco Gonzalez-Franco and Tabitha C Peck. 2018. Avatar Embodiment. Towards a Standardized Questionnaire. Frontiers in Robotics and AI 5 (2018), 74

Sandra G. Hart and Lowell E. Staveland. 1988. Development of NASA-TLX (Task Load Index): Results of Empirical and Theoretical Research. In Human Mental Workload, Peter A. Hancock and Najmedin Meshkati (Eds.). Advances in Psychology, Vol. 52 North-Holland, 139 - 183. https://doi.org/10.1016/S0166-4115(08)62386-9

Alexander Hornung, Sandip Sar-Dessai, and Leif Kobbelt. 2005. Self-calibrating optical motion tracking for articulated bodies. In IEEE Proceedings. VR 2005. Virtual Reality, 2005. IEEE, IEEE, Bonn, Germany, 75-82.
HTC. 2016. VIVE| The professional-grade VR headset. https://www.vive.com/nz/ product/

Sungchul Jung and Charles E. Hughes. 2016. The Effects of Indirect Real Body Cues of Irrelevant Parts on Virtual Body Ownership and Presence. In Proceedings of the 26th International Conference on Artificial Reality and Telexistence and the 21st Eurographics Symposium on Virtual Environments (ICAT-EGVE '16). Eurographics Association, Goslar Germany, Germany, 107-114. https://doi.org/10.2312/egve. 20161442

Sungchul Jung, Christian Sandor, Pamela J. Wisniewski, and Charles E. Hughes. 2017. RealME: The Influence of Body and Hand Representations on Body Ownership and Presence. In Proceedings of the 5th Symposium on Spatial User Interaction (SUI '17). ACM, New York, NY, USA, 3-11. https://doi.org/10.1145/3131277.3132186

Sungchul Jung, Pamela J Wisniewski, and Charles E. Hughes. 2018. In Limbo: The Effect of Gradual Visual Transition between Real and Virtual on Virtual Body Ownership Illusion and Presence. In Proceedings of the 25th IEEE Conference on Virtual Reality and 3D User Interfaces, IEEE VR 2018. IEEE, Reutlingen, Germany, 267-272.

Konstantina Kilteni, Ilias Bergstrom, and Mel Slater. 2013. Drumming in immersive virtual reality: the body shapes the way we play. IEEE transactions on visualization and computer graphics 19, 4 (2013), 597-605.

Konstantina Kilteni, Raphaela Groten, and Mel Slater. 2012. The sense of embodiment in virtual reality. Presence: Teleoperators and Virtual Environments 21, 4 (2012), 373-387.

Marc Erich Latoschik, Jean-Luc Lugrin, and Daniel Roth. 2016. FakeMi: A Fake Mirror System for Avatar Embodiment Studies. In Proceedings of the 22Nd ACM Conference on Virtual Reality Software and Technology (VRST '16). ACM, New York, NY, USA, 73-76. https://doi.org/10.1145/2993369.2993399

Marc Erich Latoschik, Daniel Roth, Dominik Gall, Jascha Achenbach, Thomas Waltemate, and Mario Botsch. 2017. The Effect of Avatar Realism in Immersive Social Virtual Realities. In Proceedings of the 23rd ACM Symposium on Virtual Reality Software and Technology (VRST '17). ACM, New York, NY, USA, Article 39, 10 pages. https://doi.org/10.1145/3139131.3139156

Ultrahaptics Ltd. 2016. Leap Motion. https://www.leapmotion.com/

Ultrahaptics Ltd. 2017. Unity, Leap Motion Developer. https://developer.leapmotion. com/unity\{\#\}5436356

Lara Maister, Mel Slater, Maria V Sanchez-Vives, and Manos Tsakiris. 2015. Changing bodies changes minds: owning another body affects social cognition. Trends in cognitive sciences 19, 1 (2015), 6-12.

MakeHuman. 2018. www.makehumancommunity.org. http://www. makehumancommunity.org/

Microsoft. 2014. Kinect - Windows app development. https://developer.microsoft. com/en-us/windows/kinect

Monoflow. 2016. UniOSC | UniOSC, the OSC solution for Unity3d. http://uniosc. monoflow.org/

Leonel Morgado, Bernardo Cardoso, Fausto de Carvalho, Luís Fernandes, Hugo Paredes, Luís Barbosa, Benjamim Fonseca, Paulo Martins, and Ricardo Rodrigues Nunes. 2015. Separating gesture detection and application control concerns with a multimodal architecture. In 2015 IEEE International Conference on Computer and Information Technology; Ubiquitous Computing and Communications; Dependable, Autonomic and Secure Computing; Pervasive Intelligence and Computing. IEEE, IEEE, Liverpool, UK, $1548-1553$.

NaturalPoint. 2019. OptiTrack - Motion Capture Systems. https://optitrack.com/

Alexander Nilsson, Ann-Sofie Axelsson, Ilona Heldal, and Ralph Schroeder. 2002. The long-term uses of shared virtual environments: An exploratory study. In The social life of a vatars. Springer, London, 112-126.

Ye Pan and Anthony Steed. 2017. The impact of self-avatars on trust and collaboration in shared virtual environments. PloS one 12, 12 (2017), e0189078.

RootMotion. 2019. Final IK - RootMotion. http://www.root-motion.com/final-ik.html

Daniel Roth, Jean-Luc Lugrin, Julia Büser, Gary Bente, Arnulph Fuhrmann, and Marc Erich Latoschik. 2016. A simplified inverse kinematic approach for embodied vr applications. In 2016 IEEE Virtual Reality (VR). IEEE, IEEE, Greenville, SC, USA, 275-276.

Daniel Roth, David Mal, Christian Felix Purps, Peter Kullmann, and Marc Erich Latoschik. 2018. Injecting Nonverbal Mimicry with Hybrid Avatar-Agent Technologies: A NaÏVe Approach. In Proceedings of the Symposium on Spatial User Interaction (SUI '18). ACM, New York, NY, USA, 69-73. https://doi.org/10.1145/3267782.3267791

Eva-Lotta Salinäs. 2002. Collaboration in multi-modal virtual worlds: comparing touch, text, voice and video. In The social life of avatars. Springer, London, UK, 172-187.

Ralph Schroeder. 2012. The social life of avatars: Presence and interaction in shared virtual environments. Springer Science \& Business Media, Berlin, Germany.

Thomas Schubert, Frank Friedmann, and Holger Regenbrecht. 2001. The experience of presence: Factor analytic insights. Presence: Teleoperators \& Virtual Environments 10,3 (2001), 266-281.

Harrison Jesse Smith and Michael Neff. 2018. Communication Behavior in Embodied Virtual Reality. In Proceedings of the 2018 CHI Conference on Human Factors in Computing Systems (CHI '18). ACM, New York, NY, USA, Article 289, 12 pages. https://doi.org/10.1145/3173574.3173863 
Bernhard Spanlang, Jean-Marie Normand, David Borland, Konstantina Kilteni, Elias Giannopoulos, Ausiàs Pomés, Mar González-Franco, Daniel Perez-Marcos, Jorge Arroyo-Palacios, Xavi Navarro Muncunill, et al. 2014. How to build an embodiment lab: achieving body representation illusions in virtual reality. Frontiers in Robotics and AI 1 (2014), 9.

Crazy Minnow Studio. 2014. SALSA LipSync. https://assetstore.unity.com/packages/ tools/animation/salsa-lipsync-suite- 148442

$\mathrm{K}$ Swinth and Jim Blascovich. 2002. Perceiving and responding to others: Human human and human-computer social interaction in collaborative virtual environments. In Proceedings of the 5th Annual International Workshop on PRESENCE, Vol. 392. ISPR, London, UK.

Unity Technologies. 2017. Unity Real-Time Development Platform |3D, 2D VR \& AR Visualizations. https://unity.com/

Geoffrey C. Urbaniak and Scott Plous. 1997. Research Randomizer. https://www randomizer.org/

Daniela Villani, Claudia Repetto, Pietro Cipresso, and Giuseppe Riva. 2012. May I experience more presence in doing the same thing in virtual reality than in reality?
An answer from a simulated job interview. Interacting with Computers 24, 4 (2012), 265-272.

Daniela Villani, Chiara Rotasperti, Pietro Cipresso, Stefano Triberti, Claudia Carissoli, and Giuseppe Riva. 2017. Assessing the emotional state of job applicants through a virtual reality simulation: a psycho-physiological study. In eHealth $360^{\circ}$. Springer, Cham, 119-126.

Steue Whittaker. 2003. Theories and Methods in Mediated Communication: Steve Whittaker. In Handbook of discourse processes. Routledge, London, UK, 246-289.

Yuanjie Wu, Yu Wang, Sungchul Jung, Simon Hoermann, and Robert W. Lindeman. 2019. Towards an articulated avatar in VR: Improving body and hand tracking using only depth cameras. Entertainment Computing 31 (2019), 100303. https: //doi.org/10.1016/j.entcom.2019.100303

Nick Yee and Jeremy Bailenson. 2007. The Proteus effect: The effect of transformed self-representation on behavior. Human communication research 33,3 (2007), 271290 